01

\title{
Черенковское трение и излучение нейтральной поляризующейся частицы при движении вблизи прозрачной диэлектрической пластины
}

\author{
(C) Г.В. Дедков, А.А. Кясов
}

Кабардино-Балкарский государственный университет, Нальчик E-mail: gv_dedkov@mail.ru

Поступило в Редакцию 10 января 2017 г.

Получены общие выражения для интенсивности излучения, силы торможения, скорости нагрева и ускорения малой поляризующейся частицы в условиях черенковского трения: при релятивистском движении параллельно поверхности гладкой толстой прозрачной диэлектрической пластины. Проводится сравнение с результатами других авторов.

DOI: 10.21883/PJTF.2017.16.44935.16705

Трение Казимира, или квантовое трение, - эффект квантовой электродинамики, возникающий при относительном движении в вакууме параллельных друг другу гладких поверхностей двух тел или при движении малой частицы параллельно поверхности протяженного тела (пластины), обусловленный квантовыми флуктуациями электромагнитного поля $[1,2]$. Процессы, связанные с квантовым и „тепловым“ (при конечной температуре тел) трением, вызывали значительный интерес в течение последних десятилетий работы (см. [3-5] и ссылки в них).

Важным следствием квантового трения является возможность генерации электромагнитного излучения при выполнении черенковского условия $V>c / n$, где $V$ - скорость частицы (здесь и далее будем рассматривать именно этот случай), $c$ - скорость света в вакууме, $n$ - показатель преломления покоящегося тела (прозрачной пластины диэлектрика) [6-8].

В соответствии с интерпретацией [7] физической причиной возникающего излучения является аномальный эффект Доплера, при котором частота квантов электромагнитного поля, поглощаемых частицей, отри- 


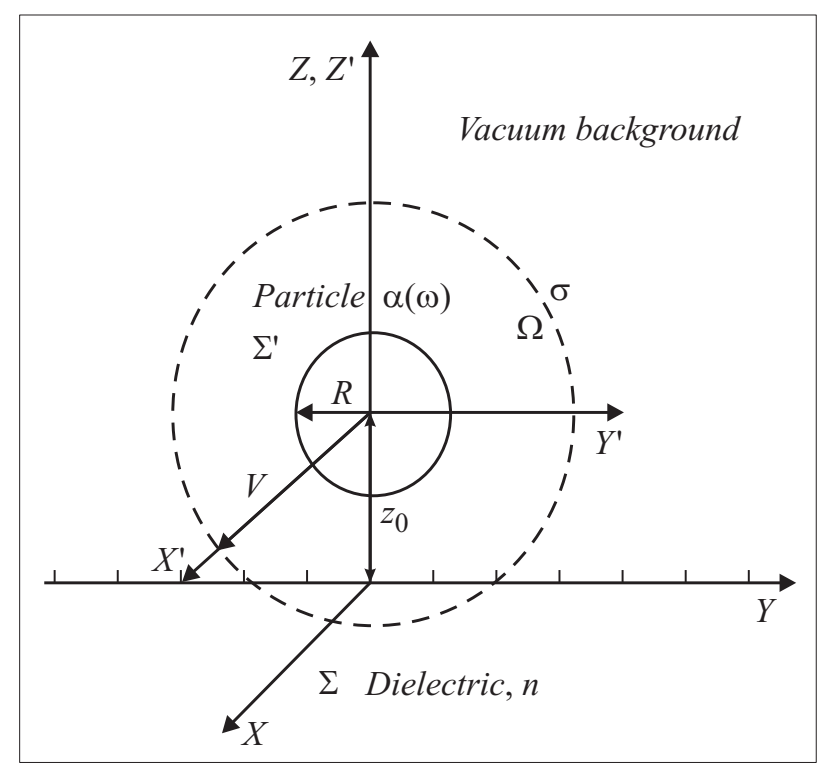

Геометрическая конфигурация и системы отсчета $\Sigma$ и $\Sigma^{\prime}$, соответствующие диэлектрической пластине и частице. Штриховой линией показана граница волновой зоны излучающей частицы.

цательна в ее системе покоя. В результате этого частица переходит в возбужденное квантовое состояние с более высокой энергией и излучает фотон в пределах черенковского конуса. В отличие от авторов работ $[7,8]$, в которых расчет интенсивности излучения проводился иначе, мы будем исходить из общих результатов нашей теории флуктуационно-электромагнитного взаимодействия релятивистской частицы, движущейся вблизи поверхности $[9,10]$. Это позволяет глубже понять электродинамическую суть проблемы и найти важные соотношения между электродинамическими, механическими и тепловыми величинами. Наше конечное выражение для интенсивности излучения совпадает с полученным в [7], но несколько отличается от [8]. По сравнению с [7] имеются некоторые отличия в соотношении между радиационной силой, мощностью излучения и скоростью изменения массы покоя в системе отсчета $\Sigma$ покоящейся пластины.

Письма в ЖТФ, 2017, том 43, вып. 16 
Используемая геометрическая конфигурация показана на рисунке. Она неоднократно применялась нами в расчетах нормальных и тангенциальных сил флуктуационно-электромагнитного взаимодействия и скорости нагрева частицы $[9,10]$. Характер основных электродинамических связей с указанными величинами определяется соотношениями

$$
\begin{gathered}
\int\langle\mathbf{j} \cdot \mathbf{E}\rangle d^{3} r=\langle\mathbf{d} \cdot \mathbf{E}+\dot{\mathbf{m}} \cdot \mathbf{B}\rangle+\mathbf{V} \cdot\left\langle\nabla(\mathbf{d} \cdot \mathbf{E}+\mathbf{m} \cdot \mathbf{B}\rangle=\dot{Q}+F_{x} V,\right. \\
\dot{Q}^{\prime}=\int_{\Omega^{\prime}}\left\langle\mathbf{j}^{\prime} \mathbf{E}^{\prime}\right\rangle d^{3} r^{\prime}=\gamma^{2}\langle\dot{\mathbf{d}} \cdot \mathbf{E}+\dot{\mathbf{m}} \cdot \mathbf{B}\rangle=\gamma^{2} \dot{Q} \\
-\frac{d W}{d t}=\oint_{\sigma} \mathbf{S} \cdot d \boldsymbol{\sigma}+\int_{\Omega}\langle\mathbf{j} \cdot \mathbf{E}\rangle d^{3} r
\end{gathered}
$$

где $\gamma-$ фактор Лоренца, $W=(1 / 8 \pi) \int_{\Omega}\left(\left\langle\mathbf{E}^{2}\right\rangle+\left\langle\mathbf{B}^{2}\right\rangle\right) d^{3} r-$ энергия поля в объеме $\Omega$, ограниченном волновой поверхностью, окружающей частицу (см. рисунок), $\mathbf{S}=(c / 4 \pi)\langle\mathbf{E} \times \mathbf{H}\rangle$ - вектор Пойнтинга, $\mathbf{d}$ и $\mathbf{m}$ - флуктуационные электрический и магнитный дипольные моменты частицы, $\mathbf{E}, \mathbf{B}, \mathbf{H}$ и $\mathbf{j}-$ флуктуационные поля и плотность электрического тока; угловые скобки и точки сверху означают квантовостатистическое усреднение и дифференцирование по времени. Все величины, не помеченные штрихом, относятся к системе отсчета $\Sigma$. В формуле (2) $\dot{Q}^{\prime}=d Q^{\prime} / d t^{\prime}-$ скорость нагрева частицы в ее системе покоя $\Sigma^{\prime}$. Заметим, что величина $\dot{Q}=d Q / d t$ имеет самостоятельный смысл и совпадает со скоростью нагрева частицы или пластины только в нерелятивистском пределе. В квазистационарном режиме $d W / d t=0$ из (3) следует

$$
I=\oint_{\sigma} \mathbf{S} \cdot d \boldsymbol{\sigma}=-\int_{\Omega}\langle\mathbf{j} \cdot \mathbf{E}\rangle d^{3} r .
$$

Кроме того, из (1) и (4) следует

$$
I=-\left(d Q / d t+F_{x} V\right) .
$$

Формулы (1)-(5) использовались нами ранее в расчетах излучения движущихся и вращающихся частиц в вакууме [11-13]. В рассматриваемой конфигурации, когда пластина прозрачна, вся приведенная 
аргументация полностью остается в силе, и можно воспользоваться общими результатами для $F_{x}$ и $\dot{Q}$, приведенными в $[9,10]$ :

$$
\begin{aligned}
F_{x}= & -\frac{\hbar \gamma}{2 \pi^{2}} \int_{0}^{\infty} d \omega \int_{-\infty}^{+\infty} d k_{x} \int_{-\infty}^{+\infty} d k_{y} k_{x} \\
& \times \sum_{i=e, m} \alpha_{i}^{\prime \prime}\left(\gamma \omega^{+}\right) \operatorname{Im}\left(\frac{\exp \left(-2 q_{0} z\right)}{q_{0}} R_{i}(\omega, \mathbf{k})\right. \\
& \times\left[\operatorname{coth}\left(\frac{\hbar \omega}{2 k_{\mathrm{B}} T_{2}}\right)-\operatorname{coth}\left(\frac{\gamma \hbar \omega^{+}}{2 k_{\mathrm{B}} T_{1}}\right)\right]+(\ldots), \\
\frac{d Q}{d t}= & \frac{\hbar \gamma}{2 \pi^{2}} \int_{0}^{\infty} d \omega \int_{0}^{\infty} d k_{x} \int_{-\infty}^{+\infty} d k_{y} \omega^{+} \\
& \times \sum_{i=e, m} \alpha_{i}^{\prime \prime}\left(\gamma \omega^{+}\right) \operatorname{Im}\left(\frac{\exp \left(-2 q_{0} z\right)}{q_{0}} R_{i}(\omega, \mathbf{k})\right) \\
& \times\left[\operatorname{coth}\left(\frac{\hbar \omega}{2 k_{\mathrm{B}} T_{2}}\right)-\operatorname{coth}\left(\frac{\gamma \hbar \omega^{+}}{2 k_{\mathrm{B}} T_{1}}\right)\right]+(\ldots),
\end{aligned}
$$

где $\omega^{+}=\omega+k_{x} V, T_{1}$ и $T_{2}$ - локальные температуры частицы (в $\Sigma^{\prime}$ ) и пластины (в $\Sigma), \alpha_{e, m}^{\prime \prime}(\omega)$ - мнимые компоненты электрической и магнитной поляризуемости, слагаемые (. . ) описывают взаимодействие с вакуумными модами электромагнитного поля (в отсутствие пластины) и не вносят вклада в последующие результаты. Вспомогательные величины в (6) и (7) определяются соотношениями

$$
\begin{aligned}
R_{e}(\omega, \mathbf{k})= & \Delta_{e}(\omega)\left[2\left(k^{2}-k_{x}^{2} \beta^{2}\right)\left(1-\omega^{2} / k^{2} c^{2}\right)+\left(\omega^{+}\right)^{2} / c^{2}\right] \\
& +\Delta_{m}(\omega)\left[2 k_{y}^{2} \beta^{2}\left(1-\omega^{2} / k^{2} c^{2}\right)+\left(\omega^{+}\right)^{2} / c^{2}\right], \\
R_{m}(\omega, \mathbf{k})= & \Delta_{m}(\omega)\left[2\left(k^{2}-k_{x}^{2} \beta^{2}\right)\left(1-\omega^{2} / k^{2} c^{2}\right)+\left(\omega^{+}\right)^{2} / c^{2}\right] \\
& +\Delta_{e}(\omega)\left[2 k_{y}^{2} \beta^{2}\left(1-\omega^{2} / k^{2} c^{2}\right)+\left(\omega^{+}\right)^{2} / c^{2}\right],
\end{aligned}
$$

Письма в ЖТФ, 2017, том 43, вып. 16 


$$
\begin{aligned}
& \Delta_{e}(\omega)=\frac{q_{0} \varepsilon(\omega)-q}{q_{0} \varepsilon(\omega)+q}, \quad \Delta_{m}(\omega)=\frac{q_{0} \mu(\omega)-q}{q_{0} \mu(\omega)+q}, \\
& q=\left(k^{2}-\left(\omega^{2} / c^{2}\right) \varepsilon(\omega) \mu(\omega)\right)^{1 / 2}, \\
& q_{0}=\left(k^{2}-\omega^{2} / c^{2}\right)^{1 / 2}, \quad k^{2}=k_{x}^{2}+k_{y}^{2} .
\end{aligned}
$$

В случае прозрачного диэлектрика $\varepsilon(\omega)=n^{2}, \operatorname{Im} \varepsilon(\omega)=0, \mu(\omega)=1$, поэтому коэффициенты $\Delta_{e, m}(\omega)$ принимают вид

$$
\begin{gathered}
\Delta_{e}(\omega)=\frac{n^{2} \sqrt{k^{2}-\omega^{2} / c^{2}}-\sqrt{k^{2}-n^{2} \omega^{2} / c^{2}}}{n^{2} \sqrt{k^{2}-\omega^{2} / c^{2}}+\sqrt{k^{2}-n^{2} \omega^{2} / c^{2}}}, \\
\Delta_{m}(\omega)=\frac{\sqrt{k^{2}-\omega^{2} / c^{2}}-\sqrt{k^{2}-n^{2} \omega^{2} / c^{2}}}{\sqrt{k^{2}-\omega^{2} / c^{2}}+\sqrt{k^{2}-n^{2} \omega^{2} / c^{2}}}
\end{gathered}
$$

Выполняя предельные переходы $T_{1} \rightarrow 0, T_{2} \rightarrow 0$ в (6), (7) с учетом соотношений $\operatorname{coth}\left(\hbar \omega / 2 k_{\mathrm{B}} T_{2}\right) \rightarrow \operatorname{sign} \omega, \operatorname{coth}\left(\hbar \omega^{+} / 2 k_{\mathrm{B}} T_{1} \rightarrow \operatorname{sign} \omega^{+}\right.$, имеем

$$
\begin{aligned}
F_{x}= & \frac{2 \hbar \gamma}{\pi^{2}} \int_{0}^{\infty} d \omega \theta(n \beta-1) \int_{\omega / V}^{n \omega / c} d k_{x} k_{x} \int_{0}^{\sqrt{n^{2} \omega^{2} / c^{2}-k_{x}^{2}}} d k_{y} \sum_{i=e, m} \alpha_{i}^{\prime \prime}\left(\gamma \omega^{-}\right) \\
& \times \operatorname{Im}\left(\frac{\exp \left(-2 q_{0} z_{0}\right)}{q_{0}} R_{i}\left(\omega,-k_{x}\right)\right), \\
\dot{Q}= & \frac{2 \hbar \gamma}{\pi^{2}} \int_{0}^{\infty} d \omega \theta(n \beta-1) \int_{\omega / V}^{n \omega / c} d k_{x} \int_{0}^{\sqrt{n^{2} \omega^{2} / c^{2}-k_{x}^{2}}} d k_{y} \omega^{-} \sum_{i=e, m} \alpha_{i}^{\prime \prime}\left(\gamma \omega^{-}\right) \\
& \times \operatorname{Im}\left(\frac{\exp \left(-2 q_{0} z_{0}\right.}{q_{0}} R_{i}\left(\omega,-k_{x}\right),\right.
\end{aligned}
$$

где $R_{i}\left(\omega,-k_{x}\right)$ совпадает с $R_{e, m}(\omega, \mathbf{k})$ при $\mathbf{k}=\left(-k_{x}, k_{y}\right)$ и $\omega^{-}=$ $=\omega-k_{x} V$. 
Используя (5), (12) и (13), находим

$$
\begin{aligned}
I= & -\frac{2 \hbar \gamma}{\pi^{2}} \int_{0}^{\infty} d \omega \theta(n \beta-1) \int_{\omega / V}^{n \omega / c} d k_{x} \int_{0}^{\sqrt{n^{2} \omega^{2} / c^{2}-k_{x}^{2}}} d k_{y} \omega \sum_{i=e, m} \alpha_{i}^{\prime \prime}(\gamma \omega) \\
& \times \operatorname{Im}\left(\frac{\exp \left(-2 q_{0} z_{0}\right)}{q_{0}} R_{i}\left(\omega,-k_{x}\right)\right)
\end{aligned}
$$

где $\theta(x)$ - функция Хевисайда. Формулы (12) и (14) совпадают с аналогичными формулами из работы [7] с тем отличием, что в (12) и (14) учтен также вклад магнитной поляризуемости $\alpha_{m}^{\prime \prime}(\omega)$ частицы. Пределы интегрирования по волновым векторам в (12)-(14) соответствуют условию аномального эффекта Доплера, поскольку в системе покоя $\Sigma^{\prime}$ частицы частота фотона отрицательна: $\omega^{\prime}=\gamma\left(\omega-k_{x} V\right)=\gamma \omega^{-}<0$. Благодаря аналитическим свойствам функций $\alpha_{e, m}^{\prime \prime}(\omega)$ и $\exp \left(-2 q_{0} z_{0}\right) / q_{0}$ из (12), (13) и (14) следует, что $F_{x}<0, \dot{Q}>0$ и $I>0$. Это означает, что именно конверсия кинетической энергии частицы в излучение является преобладающим механизмом в процессе черенковского трения (в силу (5)). Сама частица при этом нагревается (см. (2)). Заметим, что формулы (12)-(14) справедливы и при наличии дисперсии показателя преломления: $n=n(\omega)$, если считать, что частотное интегрирование выполняется по области прозрачности диэлектрической пластины, $\varepsilon^{\prime \prime}(\omega)=0$.

Кратко остановимся на отличии от результатов [7] в контексте формулы (13) и уравнения динамики частицы. Принимая во внимание (2), изменение массы покоя частицы вследствие излучения можно записать в виде

$$
\frac{d m}{d t^{\prime}}=\frac{\dot{Q}^{\prime}}{c^{2}}=\gamma^{2} \frac{\dot{Q}}{c^{2}} .
$$

Из (15) с учетом преобразования $d t=\gamma d t^{\prime}$ следует

$$
\frac{d m}{d t}=\gamma \frac{\dot{Q}}{c^{2}}
$$

Сравним этот результат с уравнением (16) в [7], которое в наших обозначениях имеет вид

$$
-V F_{x}=I+\frac{d m}{\gamma d t^{\prime}} .
$$

Письма в ЖТФ, 2017, том 43, вып. 16 
Из (17) следует

$$
\frac{d m}{d t}=-\left(V F_{x}+I\right)
$$

В отличие от (18) формула (13) в [7] для $d m / d t$ содержит дополнительный $\gamma$-фактор в правой части. При этом в соответствии с [7] $d m / d t=u^{\mu} F_{\mu}$, где $u^{\mu}$ и $F_{\mu}$ - компоненты 4-векторов скорости и силы, а из (18), (17) и (5) следует (ср. с (16))

$$
\frac{d m}{d t}=\frac{\dot{Q}}{c^{2}}
$$

Отмеченное расхождение становится существенным при $\gamma \gg 1$, т.е. именно тогда, когда интенсивность черенковского излучения максимальна, и может сказаться на решении уравнения динамики

$$
\frac{d}{d t}\left(\frac{m c \beta}{\sqrt{1-\beta^{2}}}\right)=F_{x},
$$

которое с учетом (16) приводится к виду [12]

$$
\gamma^{3} m c \frac{d \beta}{d t}=F_{x}-\beta \gamma^{2} \frac{\dot{Q}}{c}
$$

Правую часть (21) можно упростить, учитывая соотношение между силами $F_{x}$ и $F_{x}^{\prime}$, заданными в системах отсчета $\Sigma$ и $\Sigma^{\prime}$, которое получается при дифференцировании по времени преобразования Лоренца для импульса частицы

$$
F_{x}^{\prime}=F_{x}-\gamma V \frac{d m}{d t}
$$

Из (16), (21) и (22) следует

$$
\begin{gathered}
F_{x}^{\prime}=F_{x}-\beta \gamma^{2} \frac{\dot{Q}}{c}, \\
\gamma^{3} m c \frac{d \beta}{d t}=F_{x}^{\prime},
\end{gathered}
$$

Письма в ЖТФ, 2017, том 43, вып. 16 
причем явное выражение для силы $F_{x}^{\prime}$ находится при подстановке (12) и (13) в (23)

$$
\begin{aligned}
F_{x}^{\prime} & =\frac{2 \hbar \gamma^{2}}{\pi^{2}} \int_{0}^{\infty} d \omega \theta(n \beta-1) \int_{\omega / V}^{n \omega / c} d k_{x}\left(k_{x}-\beta \omega / c\right) \int_{0}^{\sqrt{n^{2} \omega^{2} / c^{2}-k_{x}^{2}}} d k_{y} \\
& \times \sum_{i=e, m} \alpha_{i}^{\prime \prime}\left(\gamma \omega^{-}\right) \operatorname{Im}\left(\frac{\exp \left(-2 q_{0} z_{0}\right)}{q_{0}} R_{i}\left(\omega,-k_{x}\right)\right) .
\end{aligned}
$$

В отличие от этого, если в уравнение динамики (20) вместо (16) подставить (19), то формула для $F_{x}^{\prime}$, получаемая с учетом (12), (13), (19) и (20), будет отличаться от (25) заменой фактора $\left(k_{x}-\beta \omega / c\right)$ в подынтегральном выражении (25) на $k_{x}\left(\gamma^{-1}+\beta^{2}\right)-\beta \omega / c$.

В заключение отметим, что полученные нами формулы (12) и (14) для силы черенковского трения и мощности излучения частицы полностью согласуются с результатами [7], полученными независимым образом, но соответствующий им вектор 4-силы, введенный авторами [7], приводит к отличию от уравнения динамики, следующего из нашего анализа. Сравнение с результатами [8] требует специального рассмотрения.

\section{Список литературы}

[1] Pendry J.B. // J. Phys.: Condens. Matter 1997. V. 9. N 47. P. 10301; New J. Phys. 2010. V. 12. P. 033028.

[2] Milton K.A., Hфye J.S., Brevik I. // Symmetry. 2016. V. 8. N 5. P. 29.

[3] Kardar M., Golestanian R. // Rev. Mod. Phys. 1999. V. 71. N 4. P. 1233.

[4] Davies P.V.W. // J. Opt. B.: Quant. Semiclass. Opt. 2005. V. 7. N 3. P. S40.

[5] Intravaia F., Henkel C., Antezza M. // Casimir physics. Lecture notes in physics, V. 834/Eds D.A.R. Dalvit, P.W. Milonni, D. Roberts, F. Da Rosa. Berlin: Springer, Ch. 11. P. 345-391.

[6] Maghrebi M.F., Golestanian R., Kardar M. // Phys. Rev. A. 2013. V. 88. P. 042515; Phys. Rev. A. 2014. V. 90. P. 012515.

[7] Pieplow G., Henkel C. // J. Phys.: Condens. Matter. 2015. V. 27. N 21. P. 214001.

[8] Волокитин А.И. // Письма в ЖЭТФ. Т. 104. В. 7. С. 534.

[9] Dedkov G.V., Kyasov A.A. // J. Phys.: Condens. Matter. 2008. V. 20. N 35. P. 354006.

Письма в ЖТФ, 2017, том 43, вып. 16 
[10] Dedkov G.V., Kyasov A.A. // Surf. Sci. 2010. V. 604. N 5-6. P. 562.

[11] Dedkov G.V., Kyasov A.A. // Phys. Scripta. 2014. V. 89. N 10. P. 105501.

[12] Dedkov G.V., Kyasov A.A. // Int. J. Mod. Phys. 2015. V. B29. N 32. P. 1550237.

[13] Дедков Г.В., Кясов А.А. // Письма в ЖТФ. 2016. Т. 42. В. 1. С. 17. 